\title{
Nonlinear Signal and Image Processing- a special issue in honour of Giovanni L. Sicuranza on his seventy-fifth birthday
}

\author{
Alberto Carini ${ }^{1 *}$, Giovanni Ramponi ${ }^{2}$ and V John Mathews ${ }^{3}$
}

This special issue on Nonlinear Signal and Image Processing serves two purposes. The first goal is to provide an overview of current trends and new research results in the broad field of Nonlinear Signal and Image Processing. The second goal is to celebrate Professor Giovanni L. Sicuranza's contributions in this field on his seventyfifth birthday. Specifically, this issue aims to honor his pioneering research in the area, his achievements, and his dedication to the EURASIP community. The guest editors of this special issue have had the good fortune and honor of working with Giovanni for more than thirty years. They have always appreciated his scientific competence and his research vision. Even more importantly, they have always enjoyed his friendship and his positive approach that make working with him most pleasant.

Professor Giovanni L. Sicuranza belongs to a distinguished group of pioneers who understood the potential and the wide domain of applications of nonlinear digital signal processing and was instrumental through his activities in developing the discipline as a mainstream research area. He has also been a strong and consistent supporter of EURASIP activities throughout his career.

Professor Sicuranza was born on September 7, 1940. After completing his education in Electronic Engineering at the University of Padova, he joined the University of Trieste (Italy) as an assistant professor of applied electronics in 1967. He remained with the University till he retired as a professor of signal and image processing in 2011. He has continued his relationship with the University of Trieste since then as a professor emeritus.

He established the Image Processing Laboratory (IPL) at the Electrical, Electronic and Computer Science Department of the University of Trieste in 1980 and was its director till 2005. Through his leadership, IPL grew into

${ }^{*}$ Correspondence: alberto.carini@uniurb.it

'DiSPeA, University of Urbino, Piazza della Repubblica, 13, 61029 Urbino, Italy

Full list of author information is available at the end of the article a preeminent research center in nonlinear signal and image processing. Many among the IPL alumni are now academic leaders in different European universities or hold leadership positions in research laboratories and industrial R\&D centers around the world.

When Professor Sicuranza started working on nonlinear signal processing, many techniques were too complex to be implemented in practical applications. Some of his early contributions aimed at devising efficient architectures for nonlinear filtering. His polynomial filter realizations using logarithmic representation or distributed arithmetic enabled the use of these filters in practical devices in the 1980s.

Professor Sicuranza's other research contributions span a wide variety of areas including multidimensional digital filters, processing of images and image sequences, image coding, nonlinear filters, polynomial filters and adaptive algorithms for system identification, echo cancellation, and active noise control. Many of the systems and nonlinear techniques he pioneered have found wide use in a number of modern DSP applications. He has published more than 200 papers in international journals and conference proceedings and eight chapters in edited books. $\mathrm{He}$ is the author or editor of four books: Multidimensional Processing of Video Signals (co-edited with S. K. Mitra, Kluwer Academic Publisher, 1992), Polynomial Signal Processing (co-authored with V J. Mathews, Wiley, 2000), Nonlinear Image Processing (co-edited with S. K. Mitra, Academic Press, 2001), and Advances in Nonlinear Signal and Image Processing, (co-edited with S. Marshall, Hindawi Publishing Corporation, 2006.).

Professor Sicuranza was a member of the technical committees of numerous international conferences, the Chairman of the VIII European Signal Processing Conference, EUSIPCO-96 (Trieste, Italy, 1996) and the Sixth Workshop on Nonlinear Signal and Image Processing, NSIP-03, (Grado, Italy, 2003). He was also the Co-chair of the IEEE International Conference on Image Processing, ICIP-05 
(Genova, Italy, 2005) and Honorary Chair of the 6th Int'l Symposium on Signal and Image Processing and Analysis, ISPA 2013 (Trieste, Italy, 2013). He was a member of the editorial board of the EURASIP journal "Signal Processing" and of the IEEE Signal Processing Magazine. He is presently an Associate Editor of "Multidimensional Systems and Signal Processing" and a member of the editorial board of the Springer journal "Signal, Image and Video Processing". Professor Sicuranza has served as a project manager of ESPRIT and COST research projects funded by the European Commission and as a consultant to several companies. He was a member of the IMDSP Technical Committee of the IEEE Signal Processing Society and a member of the Administrative Committee and Awards Chairman of EURASIP. Professor Sicuranza received the EURASIP Meritorious Service Award and the EURASIP Certificate of Merit in 2006 and 2015, respectively.

For this special issue, we received 21 articles, from which 9 were accepted. The topics range from nonlinear filter theory to nonlinear filter applications. The possible applications of proposed filters cover different fields from audio processing to image processing, video processing, and sonar.

The first article, entitled "Robust compressive sensing of sparse signals: a review," by R. E. Carrillo, A. B. Ramirez, G. R. Arce, K. E. Barner, and B. M. Sadler, overviews robust nonlinear reconstruction strategies for sparse signals based on replacing the commonly used $\ell_{2}$ norm by $\mathrm{M}$-estimators as data fidelity functions in compressed sensing. The methods discussed offer a robust framework for compressed sensing: they are particularly fitted for impulsive environments, where they outperform existing compressed sensing techniques, and they achieve good performance in light-tailed environments.

The second article in this special issue is "Generalized Hampel Filters," by R. K. Pearson, Y. Neuvo, J. Astola, and M. Gabbouj. The Hampel filter is a member of the class of decision filters and may be regarded as an extension of the median filter. The Hampel filter replaces the central value in the data window with the median if it lies far enough from the median to be deemed an outlier. This paper explores generalizations of Hampel filters obtained by applying the median filter extensions, such as the weighted Hampel filters, recursive Hampel filters, and their cascades.

The third article, "Self-organizing kernel adaptive filtering," by S. Zhao, B. Chen, Z. Cao, P. Zhu, and J. C. Principe, deals with kernel adaptive filters and presents a model-selection strategy based on minimum description length that keeps the kernel least-mean-square model tuned to the complexity of the input data. The kernel adaptive filter presented in this paper adapts its model order as well as its coefficients online, behaving as a self-organizing system and achieving a good compromise between system accuracy and computational complexity without a priori knowledge.

In significance-aware filtering, the estimation of a nonlinear echo path is decomposed into beneficially interacting subsystems, each of which can be adapted with high computational efficiency. The significanceaware Hammerstein group models decompose the nonlinear acoustic echo path into a direct-path part, modeled by a Hammerstein group model and a complementary part, modeled by an efficient Hammerstein model. In the fourth article, "Significance-aware filtering for nonlinear acoustic echo cancellation," by C. Hofmann, C. Huemmer, M. Guenther, and W. Kellermann, the authors propose a novel equalization-based significance-aware structure, where the echo path is equalized by a linear filter to allow for an estimation of the loudspeaker nonlinearities by very small and efficient models.

The fifth article, "Introducing oriented Laplacian diffusion into a variational decomposition model," by $\mathrm{R}$. Shahidi and C. Moloney improves the image decomposition model proposed by Osher, Solé, and Vese in 2003. The paper introduces diffusion with an oriented Laplacian for oriented texture in lieu of the usual Laplacian operator used to solve the Osher, Solé, and Vese model. This significantly reduces the presence of high-frequency texture in the noise component. The experimental results consider test images with oriented texture and compare the oriented Laplacian model with the Osher, Solé, and Vese model and with the mean curvature model. They show that the oriented Laplacian model yields higher signalto-noise ratios or at least visually superior denoising performances than the other competing methods.

A classic approach for reducing the computational complexity of Volterra filters defines a matrix of filter coefficients and applies the singular value decomposition to the matrix. Implementations based on discarding the smaller singular values effectively reduces the complexity of Volterra filters. This approach is very effective for second-order Volterra filters, but its extension to higherorder Volterra filters is not straightforward. In "A reducedrank approach for implementing higher-order Volterra filters," by E. L. O. Batista and R. Seara, the authors develop a novel reduced-rank approach for the implementation of higher-order Volterra filters. Their approach is based on the decomposition of the higher-order Volterra kernels into structures composed only of second-order kernels. Applying the singular value decomposition to the coefficient matrices of these second-order kernels, effective implementations of higher-order Volterra filters are obtained.

In "A procedure to locate the eyelid position in noisy videokeratoscopic images," T. Schäck, M. Muma, W. Alkhaldi, and A. M. Zoubir proposes a novel procedure to robustly determine the eyelid position in high-speed 
videokeratoscopic images. The eyelid position is an important information in such images, since the eyelids affect the state of the cornea and the tear film dynamics. The very low contrast of videokeratoscopic images and the occlusions caused by the eyelashes cause difficulties in solving this problem. In this paper's approach, nonlinear image filtering is first performed to remove the eyelashes. Then, an image segmentation approach based on morphological operations and active contours is used to provide a set of candidate pixels. A verification procedure reduces the set to those pixels that are likely to contribute to accurately locate the eyelid edge. Finally, the authors use robust $\mathrm{M}$-estimation to fit a parametric model to the selected candidate pixels.

In "Object representation for multi-beam sonar image using local higher-order statistics," H. Li, J. Gao, W. Du, T. Zhou, C. Xu, and B. Chen investigates how high-order statistics are associated with the presence of an object in an image. In the paper's approach, an image of the skewness of the data is obtained using a sliding window. When objects are present in the image, a relation is found between the size of the object in the region of interest, the SNR, and the values of the skewness. Experiments with real-world sonar images showed that local skewness is a robust object representation, even in the presence of strong variations of SNR between adjacent frames.

The last article, "Static force field representation of environments based on agents' nonlinear motions," by D. Campo, A. Betancourt, L. Marcenaro, and C. Regazzoni, deals with the analysis of trajectories followed by agents moving in an environment and with the detection of abnormalities in such trajectories. The authors exploit a parametric model in which crowd motion is represented as the effect of forces interacting among the agents. A bank of Kalman filters evolve from an initial random walk state, basing on the agents' actual motion. This model can provide results when detailed information about the environment is not available, but the position of the agents can be determined. The estimated positions incrementally reveal the characteristics of the environment, such as the presence, the location, the intensity, and the action range of attractive areas.

As guest editors of this special issue, we are grateful to the 45 reviewers who helped rigorously vet the papers and helped us select the contents of this issue. We once again express our appreciation for Professor Sicuranza's contributions in this field as well as for his friendship. It has been our immense pleasure to honor him through this compilation of articles in the area he pioneered.

\section{Publisher's Note}

Springer Nature remains neutral with regard to jurisdictional claims in published maps and institutional affiliations.

\section{Author details}

${ }^{1}$ DiSPeA, University of Urbino, Piazza della Repubblica, 13, 61029 Urbino, Italy. ${ }^{2}$ DIA, University of Trieste, Via A. Valerio 10, 34127 Trieste, Italy. ${ }^{3}$ School of Electrical Engineering and Computer Science, Oregon State University, Corvallis, OR 97331, USA.

Received: 3 March 2017 Accepted: 7 March 2017

Published online: 11 April 2017

\section{Submit your manuscript to a SpringerOpen ${ }^{\circ}$ journal and benefit from:}

- Convenient online submission

- Rigorous peer review

- Immediate publication on acceptance

- Open access: articles freely available online

- High visibility within the field

- Retaining the copyright to your article

Submit your next manuscript at $\boldsymbol{\triangleright}$ springeropen.com 